\title{
Una nueva mirada al arte suntuario medieval hispánico
}

\author{
Francisco de Asís García García \\ Universidad Complutense de Madrid \\ Departamento de Historia del Arte I (Medieval) \\ fdagarcia@ghis.ucm.es
}

\section{RESUMEN}

Como ensayo introductorio, se revisan cuestiones de actualidad en el estudio de las artes suntuarias medievales a fin de enmarcar las contribuciones recogidas en este volumen en diversas problemáticas histórico-artísticas.

Palabras clave: Artes suntuarias, arte medieval hispánico, historiografía, vías de estudio.

\section{A New Look at Medieval Hispanic Sumptuary Arts}

\begin{abstract}
As an introductory essay, this article reviews current issues in the study of medieval sumptuary arts in order to frame the articles collected in this volume around contemporary art-historical debates.
\end{abstract}

Key words: Sumptuary Arts, Medieval Hispanic Art, Historiography, Perspectives of Study. 
En los últimos años, exposiciones de repercusión internacional, reuniones científicas, proyectos de investigación e iniciativas museológicas han situado a las artes suntuarias medievales en un lugar privilegiado dentro de los debates histórico-artísticos. Asimismo, la aplicación de las nuevas tecnologías a su estudio ha repercutido en una decisiva renovación metodológica y en el planteamiento de líneas de investigación alternativas para su análisis. La consideración de lo suntuario desde los postulados de la antropología histórica y su apreciación desde campos como la historia económica y social han permitido abordar el mundo del objeto con unas miras más amplias que enriquecen el discurso de la historia del arte ${ }^{1}$.

Recogiendo dichas aportaciones, asumidas como punto de partida, este volumen centra su atención en las artes suntuarias de la Edad Media hispánica con el fin de proyectar sobre estas una nueva mirada desde múltiples perspectivas: su diversa estima en la cultura medieval; su contribución al imaginario simbólico; el uso de los objetos al servicio del culto y de la expresión del poder; el valor de los materiales y la diversidad técnica en la creación artística; la circulación de piezas y el fenómeno coleccionista; y la contribución de la museología a la valoración de los objetos en nuevos marcos expositivos y a la creación de nuevos discursos.

Los debates en torno al estatus de las artes suntuarias y su relación jerárquica con otras manifestaciones artísticas constituyen un lugar común en la historiografía ${ }^{2}$. Las polémicas afloran al cuestionarse la nomenclatura empleada para su definición: ¿decorativas? ¿menores? ¿aplicadas? ¿industriales? Son términos que proyectan una mirada anacrónica, sesgada e incompleta sobre una realidad mucho más compleja, especialmente en el periodo medieval, en el que primaban la integración y diálogo entre las artes -sin que se produjera una segregación efectiva- y donde no se aplicaban tales categorizaciones. La inclusión de los objetos suntuarios dentro del vasto concepto de cultura material se presta a una ampliación de perspectivas al incidir en el componente antropológico y social, pero corre el riesgo de subestimar otros aspectos clave -quizá más familiares para el historiador del arte-como el patrocinio, la exclusividad y la inventiva, el rango y la poética de los materiales o la dimensión significante de las obras como vehículo de expresión icónica³

Iniciativas recientes han contribuido a replantear algunas de estas cuestiones ${ }^{4}$, si bien con escasa presencia de lo hispánico en sus debates. Así, frente a centros preemi-

1 Recientes coloquios constituyen una buena muestra de la orientación epistemológica señalada: L. FELLER y A. RODRIGUEZ (dirs.), Objets sous contrainte. Circulation et valeur des choses au Moyen Âge, París, 2013; y Mercats del luxe, mercats de l'art. La Corona d'Aragó i la Mediterrània en els segles XIV i XV, Valencia, 2015 (en prensa).

2 B. BUETTNER, "Towards a Historiography of the Sumptuous Arts", C. RUDOLPH (ed.), A Companion to Medieval Art: Romanesque and Gothic in Northern Europe, Malden-Oxford-Carlton, 2006, pp. 466-487; X. BARRAL I ALTET, "Des arts mineurs qui sont majeurs", Contre l'art roman? Essai sur un passé réinventé, París, 2006, pp. 146-156.

3 Por una necesaria integración e impregnación metodológica entre disciplinas aboga M. YONAN, "Toward a Fusion of Art History and Material Culture Studies", West 86th. A Journal of Decorative Arts, Design History, and Material Culture, 18, 2 (2011), pp. 232-248.

4 Como el congreso celebrado los días 17 y 18 de marzo de 2011 por el Index of Christian Art cuyas actas se publicaron al año siguiente: C. HOURIHANE (ed.), From Minor to Major: The Minor Arts in Medieval Art History, Princeton-University Park, 2012. 
nentes y considerados como tal sin género de dudas por la historiografía canónica París, Constantinopla, Limoges, el Valle del Mosa o Colonia, por citar algunos de los más significativos-, otros ámbitos "periféricos" para los discursos histórico-artísticos dominantes han sido soslayados, careciendo del impacto crítico internacional que merecen y de acomodo en los compendios al uso ${ }^{5}$. Una marginalidad historiográfica paliada por la presencia de algunas obras emblemáticas del arte medieval hispano en colecciones de renombre que les han granjeado una privilegiada plataforma de difusión ${ }^{6}$. La Península Ibérica, por su enclave geográfico y devenir histórico en los siglos medievales, aporta un singular muestrario al arte suntuario en el que se dan cita corrientes europeas de vanguardia junto a la creatividad andalusí y los estímulos de Bizancio canalizados por el Mediterráneo. Basta recordar los productos que circulaban en León en el s. $\mathrm{X}^{7} \mathrm{o}$ los gravados por el arancel pirenaico instituido por los reyes de Pamplona y Aragón en la centuria siguiente ${ }^{8}$ para adquirir consciencia del terreno abonado que suponían en este sentido los reinos hispanos, un crisol que el desarrollo económico y la fluidez de los intercambios de la Baja Edad Media potenciaron más aún.

Como historiadores del arte, la mirada coetánea a las obras que estudiamos resulta de especial valía a la hora de estimar su verdadero papel para quienes las crearon y dieron uso en las sociedades medievales, de modo que toda reflexión sobre el estatus de lo suntuario ha de anclarse en el conocimiento más preciso posible de los contextos de origen. La aportación de Laurent Feller sobre la valoración de los objetos de lujo en ambientes como la corte y en procesos como las transferencias de patrimonio o los intercambios comerciales presta un apropiado marco metodológico y conceptual para la comprensión de los aspectos tratados a lo largo del volumen.

Una proporción significativa de los artículos reunidos incide en el carácter distintivo de las producciones suntuarias en el marco de la creación artística, la dimensión simbólica de los objetos y los usos y funciones que desempeñaban. En determinados contextos el valor de las piezas iba más allá de su cualidad material y se veía particularmente enfatizado por su capacidad de remitir a realidades abstractas o entrar en contacto con lo sagrado. Es evidente el caso de las regalia y todo aquel elemento que encarnaba el poder ${ }^{9}$, así como el de los relicarios, cuyo estudio ha gozado de parti-

5 La problemática de la centralidad y de la geografía artística, planteada para el estudio de las dinámicas creativas en el ensayo fundacional de E. CASTELNUOVO y C. GINZBURG, "Centro e periferia", G. PREVITALI (ed.), Storia dell'arte italiana, vol. I, Turín, 1979, pp. 285-352, ha sido recientemente sondeada en lo referente a la posición del historiador y su discurso: P. PIOTROWSKI, "On the Spatial Turn, or Horizontal Art History", Umění, 56, 5 (2008), pp. 378-383.

6 Caso de publicaciones como las de J. BECKWITH, Caskets from Cordoba, Londres, 1960, y M. ROSSER-OWEN, Islamic Arts of Spain, Londres, 2010, o la de X. DECTOT, Céramiques hispaniques. XII ${ }^{2}-$ XVII siècle, París, 2007, elaboradas a propósito de los fondos del Victoria \& Albert Museum de Londres y del Musée national du Moyen Âge de París, respectivamente.

7 C. SÁNCHEZ ALBORNOZ, Una ciudad de la España cristiana hace mil años. Estampas de la vida en León, Madrid, 1966 (5 ed.), pp. 32-33, 65-67, 103-105 y 125-128.

8 J.M. LACARRA, "Un arancel de aduanas del siglo XI", Actas del Primer Congreso Internacional de Estudios Pirenaicos, Zaragoza, 1952, pp. 21-36.

9 I.G. BANGO TORVISO, "De las insignias reales en la España medieval", M.L. MELERO MONEO et al. (eds.), Imágenes y promotores en el arte medieval. Miscelánea en homenaje a Joaquín Yarza Luaces, 
cular éxito en las últimas décadas ${ }^{10}$. Sin duda la funcionalidad litúrgica de muchas realizaciones suntuarias contribuyó también a enaltecer su estatus al desempeñar un papel mediador en el diálogo con lo sobrenatural. El ajuar litúrgico y los espacios activados en la celebración sacramental vertebran el discurso de María Luisa Martín en torno al Bautismo y la Unción, mientras que dos objetos ligados al culto eucarístico y martirial centran la investigación de Manuel Parada y Almudena Cros, quienes abordan las obras a través de los perfiles del promotor y del artista que intervinieron en su confección. La devoción a la Eucaristía impulsó la realización de cuantiosos receptáculos de orfebrería en el tardomedievo y la Edad Moderna, como expone Ana Sousa al estudiar algunas piezas portuguesas caracterizadas por un bilingüismo formal. Contextos ceremoniales de otra índole, dentro de la esfera secular, prestan el marco a Laura Vegas y Teresa Viñas para el censo y estudio de piezas al servicio de la exaltación sensorial en el cuatrocientos castellano.

En los últimos tiempos una de las líneas que mayor repercusión ha obtenido en el estudio de las artes suntuarias es la investigación sobre los tesoros ${ }^{11}$. El análisis de la formación y composición de estos conjuntos como entidad, así como de las piezas individuales que los conforman, se ha plasmado en recientes volúmenes de autoría colectiva $^{12}$ y en obras que han abordado monográficamente casos particulares ligados a determinados personajes o instituciones ${ }^{13}$. En el ámbito hispano ha de mencionarse como hito de singular relevancia, por su planteamiento y la nómina de obras congregadas, la exposición celebrada en San Isidoro de León entre diciembre de 2000 y febrero de 2001 Maravillas de la España medieval. Tesoro sagrado y monarquía ${ }^{14}$.

Bellaterra, 2001, pp. 59-66; y M. SERRANO COLL, "Los signos de poder: regalias como complemento a los emblemas de uso inmediato", Emblemata, 17 (2011), pp. 129-154.

10 Desde el clásico de M.-M. GAUTHIER, Les routes de la foi. Reliques et reliquaires de Jérusalem à Compostelle, Friburgo (Suiza), 1983, a los más recientes The Way to Heaven: Relic Veneration in the Middle Ages, catálogo de la exposición (Ámsterdam-Utrecht, 2000-2001), Baarn, 2000; B. REUDENBACH y G. TOUSSAINT, Reliquiare im Mittelalter, Berlín-Hamburgo, 2005; Treasures of Heaven: Saints, Relics and Devotion in Medieval Europe, catálogo de la exposición (Cleveland-Baltimore-Londres, 2010-2011), Londres, 2011; y C. HAHN, Strange Beauty. Issues in the Making and Meaning of Reliquaries, 400 -circa 1204, University Park, 2012. Un balance de las investigaciones en P. CORDEZ, "Les reliques, un champ de recherches. Problèmes anciens et nouvelles perspectives", Bulletin d'information de la Mission Historique Française en Allemagne, 43 (2007), pp. 102-116.

${ }_{11}$ M. TOMASI, “Des trésors au Moyen Âge : enjeux et pratiques, entre réalités et imaginaire", Perspective (2009-1), pp. 137-141.

12 E.M. TYLER (ed.), Treasure in the Medieval West, Woodbridge-Rochester, 2000; S. GELICHI y C. LA ROCCA (eds.), Tesori. Forme di accumulazione della ricchezza nell'alto medioevo (secoli V-XI), Roma, 2004; L. BURKART et al., Le trésor au Moyen Âge. Questions et perspectives de recherche / Der Schatz im Mittelalter. Fragestellung und Forschungsperspektiven, Neuchâtel, 2005; L. BURKART et al. (eds.), Le trésor au Moyen Âge. Discours, pratiques et objets, Florencia, 2010; y Les trésors des églises à l'époque romane, número monográfico de Les Cahiers de Saint-Michel de Cuxa, XLI (2010).

13 P. CORDEZ (ed.), Charlemagne et les objets. Des thésaurisations carolingiennes aux constructions mémorielles, Berna et al., 2012; y los catálogos de las exposiciones The Treasury of San Marco. Venice / Le trésor de Saint-Marc de Venise, Milán, 1984; Le trésor de Saint-Denis, París, 1991; Le trésor de la SainteChapelle, París, 2001; The Treasury of Basel Cathedral, Nueva York, 2001; Le trésor de Conques, París, 2001; Medieval Treasures from Hildesheim, Nueva York, 2013; y Le Trésor de l'abbaye de Saint-Maurice d'Agaune, París, 2014.

14 I.G. BANGO TORVISO (dir. cient.), Maravillas de la España medieval. Tesoro sagrado y monarquía, catálogo de la exposición (León, 2000-2001), 2 vols., 2001. 
Como pudo ponerse de relieve en la muestra leonesa, el tesoro sagrado de la monarquía asturiana constituye uno de los capítulos más significativos del arte suntuario medieval hispano, y no es de extrañar que atrajera la atención de la comunidad científica internacional décadas atrás ${ }^{15}$. Con todo, a diferencia del éxito historiográfico de piezas emblemáticas como las cruces de los Ángeles o de la Victoria, la Arqueta de las Ágatas no ha suscitado el interés que merece una obra de su categoría. La contribución de César García de Castro viene a colmar este vacío con un exhaustivo análisis. Asimismo, frente a casos como el de la Cámara Santa de Oviedo o el de otras monarquías hispánicas en fechas más recientes del periodo medieval, el tesoro de la primera dinastía regia portuguesa nos es menos conocido, en parte por la escasez de los bienes conservados y de las fuentes que los registran. Su composición y evolución son objeto del estudio firmado por Ana Maria Rodrigues.

El relativo estatismo o carácter arcaizante de algunas producciones áulicas, donde la fidelidad a la tradición no es un mero factor de la creación artística sino que conlleva un mensaje de autoridad, desaparece en ámbitos como el del atuendo, avivado por una constante innovación. Con la Corona de Aragón como escenario analiza este proceso Juan Vicente García Marsilla, dando cabida a fenómenos interrelacionados como la demanda, la producción y el consumo. La atracción por la novedad y su incidencia en ámbitos cortesanos es explorada por Catarina Simões para el contexto portugués de los Descubrimientos, ampliando el concepto de lo suntuario a toda una serie de bienes preciados caracterizados por el exotismo de su apariencia u origen. $\mathrm{La}$ mirada arqueológica de Alexandra Uscatescu nos traslada de lo áulico a registros más populares interrogándose por la recepción del contenido icónico de ciertos elementos de ajuar en la Castilla del s. XIII.

Junto al hecho de la tesaurización anteriormente aludido, el fenómeno del coleccionismo permite reflexionar sobre prácticas en las que las artes suntuarias han ocupado tradicionalmente un destacado lugar ${ }^{16}$. No toda reunión de objetos artísticos constituye una colección, término cuya conceptualización actual no encuentra fácilmente paralelos adecuados en el mundo medieval, donde primó lo acumulativo al margen de un orden sistemático y de un discurso definido. Con todo, las cortes principescas de finales de la Edad Media desarrollaron fórmulas en las que se han reconocido algunos fundamentos del coleccionismo moderno.

A través de una cuidadosa exploración documental y cronística, varios artículos revisan pautas y actuaciones en relación con el acopio de obras, la producción de objetos de lujo generada en torno a una determinada figura y sus avatares, y la medida en la que un personaje imponía o no una línea concreta en su entorno suntuario. Sumándose a reflexiones en este sentido presentes en buena parte de las contribuciones aquí reunidas, dos artículos indagan con particular atención en estos aspectos: Jacobo Vidal se detiene en las piezas suntuarias en la cámara de María de Castilla y sus artífices, perfilando la actividad de la reina como usuaria y promotora artística,

15 Véase la reedición de los estudios clásicos de Schlunk y Elbern sobre las obras orfebres de Oviedo llevada a cabo por C. GARCÍA DE CASTRO VALDÉS (ed.), Estudios sobre la orfebrería del Reino de Asturias, Oviedo, 2008.

16 P.A. MARIAUX, “Collecting (and Display)”, C. RUDOLPH (ed.), op. cit., 2006, pp. 213-232. 
mientras que Fernando Villaseñor pasa revista a los bienes del condestable castellano Álvaro de Luna para mostrar las altas cotas de opulencia alcanzadas por miembros de la nobleza. Con la figura de Catalina de Aragón como referente en las relaciones anglo-españolas, Emma Cahill rastrea la presencia e impacto suntuario de lo hispano en la Inglaterra Tudor.

El estudio del arte suntuario medieval se enfrenta de forma especialmente manifiesta a la pérdida de obras esenciales para el establecimiento de corpus y a la ausencia de objetos cuya relevancia o carácter distintivo no los guardaron de un malogrado fin ${ }^{17}$. Ante piezas que no han llegado hasta nosotros, pero cuya memoria ha sido preservada, la aproximación al documento es decisiva. Los metales preciosos han resultado particularmente afectados por el paso del tiempo, sometidos a vicisitudes por todos conocidas. El vaciado documental aportado en los artículos de Joan Duran-Porta y Anna Molina supone un ejercicio necesario en la reconstrucción del pasado, sea para recomponer un amplio elenco de objetos -como el de la orfebrería catalana del románico consignada en los diplomas- o para acercarnos a piezas singulares como el célebre Castell d'Amor de Pedro el Ceremonioso y los autómatas de inspiración literaria. La documentación permite no solo reconstruir las obras y registrar su existencia, sino también ilustrar sobre el mercado, la clientela y el gusto que determinaron el consumo de los objetos, como pone de relieve Miquel Àngel Capellà a propósito del Reino de Mallorca.

En otras ocasiones, es la obra suntuaria conservada la que aporta claves para la restitución de un monumento perdido y su entramado de influencias artísticas, tal y como propone Laura Molina ante los simulacros de la Capilla Real de Sevilla. En el terreno edilicio, el prestigio y consideración gozados por las telas ricas permiten a Juan Carlos Ruiz disertar sobre las relaciones entre lo suntuario y lo monumental y percibir a través de la piedra y sus revestimientos el recuerdo del pasajero boato textil. La rejería arquitectónica, en cambio, tendió a perpetuar su prominente inserción en el espacio edificado gracias a la índole de sus materiales y merced a su propia funcionalidad. Como muestra de lo señalado, Herbert González analiza los ejemplares de la parroquia de la Asunción de Barco de Ávila.

Uno de los rasgos característicos del arte suntuario es su potencial movilidad ${ }^{18}$. Esta podía verse motivada por los designios de los promotores al prever destinos para las obras, ser fomentada con fines comerciales, o incluso producirse forzadamente como resultado de acontecimientos de variado signo ${ }^{19}$. Al mismo tiempo, el tránsito de manufacturas posibilitaba su enriquecimiento y mutación de significados, y las

17 Como reflexión de carácter general acerca de dichos condicionantes véase C. TREFFORT, "Le corpus du chercheur, une quête de l'impossible? Quelques considérations introductives", Annales de Janua, 2 (2014), http://annalesdejanua.edel.univ-poitiers.fr/index.php?id=725 (último acceso 21/6/2014).

$18 \mathrm{Si}$ bien circunscritas a las relaciones interculturales, son de interés las reflexiones sobre esta cualidad expuestas en E.R. HOFFMAN, "Pathways of Portability: Islamic and Christian interchange from the tenth to the twelfth century", Art History, 24, 1 (2001), pp. 17-50, y C. SCHMIDT ARCANGELI y G. WOLF (eds.), Islamic Artefacts in the Mediterranean World. Trade, Gift Exchange and Artistic Transfer, Venecia, 2011.

19 Sobre estos mecanismos véase P. GEARY, "Sacred commodities: the circulation of medieval relics", A. APPADURAI (ed.), The social life of things. Commodities in cultural perspective, Cambridge, 1992, pp. 169-191. 
facultaba para construir socialmente su valor ${ }^{20}$. Si bien fenómenos como el expolio contribuyeron al flujo de objetos preciados en el periodo medieval, el ofrecimiento de presentes constituyó una privilegiada modalidad de circulación de obras suntuarias. Con el fin de profundizar en estos usos, Noelia Silva se adentra en la elitista eboraria califal cordobesa y examina las implicaciones de los objetos de lujo y su aparato icónico en la práctica del regalo. Por su parte, Sila Oreja recurre a las crónicas para caracterizar la dimensión ostentativa y propagandística del textil en la Castilla trastámara, valores implícitos en el obsequio de dichas obras. Como se aprecia en el texto elaborado por Joan Domenge, algunas de las joyas de carácter emblemático vinculadas con Alfonso el Magnánimo sirvieron a propósitos similares, fuera en calidad de concesión regia o de dádiva recibida por el propio monarca. En cualquier caso, se trataba de una producción exclusiva con unos canales de difusión restringidos. Sin embargo, la Baja Edad Media conoció cauces alternativos para la propagación de lo suntuario en forma de manufacturas estandarizadas ${ }^{21}$, un fenómeno ya preludiado en el s. XII por los inicios del opus lemovicense que tuvo en los marfiles parisinos o los vidrios venecianos algunas de sus producciones más conocidas. Los alabastros ingleses gozaron de una notable movilidad por su destino a la exportación, amoldándose a unas pautas comunes que no invalidan su interés iconográfico, como pone de manifiesto Fernando Pérez al inspeccionar la temática jacobea presente en varias de estas piezas.

La especificidad de las artes suntuarias ha favorecido una especial cercanía de sus estudiosos a la materialidad y técnica de ejecución de las obras, aspectos que en otras parcelas de la disciplina histórico-artística se han visto ocasionalmente desplazados por el interés concedido a lo visual ${ }^{22}$. Hoy la atención a la condición matérica de los objetos como punto de partida para escrutar la dimensión estética, simbólica o económica implicada en el hecho artístico rezuma actualidad dentro de los llamados "nuevos materialismos" 23 . No debe olvidarse que técnica y materia son elementos claramente presentes en la reflexión medieval sobre lo suntuario y sus procesos crea-

20 L.E. SAURMA-JELTSCH y A. EISENBEIß (eds.), The Power of Things and the Flow of Cultural Transformations, Berlín, 2010.

21 Acerca de ambas modalidades de ejecución véase O. PÉREZ MONZÓN, "Producción artística en la Baja Edad Media. Originalidad y/o copia”, M.V. CHICO PICAZA, L. FERNÁNDEZ FERNÁNDEZ y M. MIQUEL JUAN (eds.), El siglo XV hispano y la diversidad de las artes, número especial de Anales de Historia del Arte, 22 (2012), pp. 85-121.

22 Si bien no faltan medievalistas que han reivindicado la dimensión física de la imagen: J.-C. BONNE, "Entre l'image et la matière: la choséité du sacré en Occident", J.-M. SANSTERRE y J.-C. SCHMITT (eds.), Les images dans les sociétés médiévales. Pour une histoire comparée, Bruselas-Roma, 1999, pp. 77-111; J. BASCHET, “L’image-objet”, L’iconographie médiévale, París, 2008, pp. 25-64 (especialmente pp. 33-39).

23 C.W. BYNUM, Christian Materiality: An Essay on Religion in Late Medieval Europe, Nueva York, 2011. Las implicaciones de la materialidad en el arte medieval centran el primer capítulo de H.L. KESSLER, Seeing Medieval Art, Peterborough-Orchard Park, 2004, pp. 19-42; del mismo autor, "The Object as Subject in Medieval Art", The Haskins Society Journal, 23 (2011), pp. 205-228. A estas cuestiones se han dedicado recientes números monográficos de Gesta, 51/1 (2012): A. KUMLER y C.R. LAKEY (eds.), Res et significatio. The Material Sense of Things in the Middle Ages, y de Different Visions. A Journal of New Perspectives on Medieval Art, 4 (2014): Active Objects (http://differentvisions.org/issue-four/, último acceso 15/4/2014). Véanse también a modo de ejemplo algunos de los títulos recogidos en I. WEINRYB, "Living Matter: Materiality, Maker, and Ornament in the Middle Ages”, Gesta, 52 (2013), pp. 113-114, n. 2. 
$\operatorname{tivos}^{24}$. Las referencias a la pureza del material y la percepción de las calidades, por citar algunos aspectos, son una constante en las fuentes literarias y se invocan, a su vez, en las prescripciones de los contratos artísticos. Desde el punto de vista de la praxis suntuaria y de su estudio, la materia prima determina y demanda un conocimiento técnico preciso en la ejecución de un objeto, a la par que condiciona las vías de análisis de las piezas e impone un marco interpretativo sensible a la propia tradición artística de ese material.

En la confluencia de aproximaciones que encaran el arte suntuario medieval desde dichos fundamentos ocupa un relevante lugar la perspectiva tecnológica. En las últimas décadas la aplicación de nuevas técnicas de estudio, con una sustancial aportación de los análisis de laboratorio, ha revolucionado la investigación de las artes suntuarias permitiendo aquilatar cronologías, reconsiderar filiaciones, reconstruir procesos creativos y, en definitiva, actualizar nuestra mirada sobre los testimonios del pasado a partir de bases más sólidas ${ }^{25}$. Someter las piezas a examen a partir de modernas tecnologías posibilita un privilegiado conocimiento de las técnicas y procesos de fabricación y su parangón con las fórmulas consignadas en la tratadística medieval. Una clara muestra de la aludida renovación metodológica es la revisión crítica de destacadas obras de orfebrería a partir de los avances producidos en el campo de la arqueometalurgia. Los conjuntos de Guarrazar y Torredonjimeno, examinados por Óscar García-Vuelta y Alicia Perea, se han beneficiado de las nuevas técnicas y herramientas de caracterización, cuya aplicación a ambos tesoros ha supuesto un sustancial avance en el conocimiento de la orfebrería visigoda.

Como han demostrado iniciativas de esta índole, el análisis tecnológico no es un fin en sí mismo, sino que resulta fundamental incardinar el estudio de la técnica y los resultados de los procedimientos analíticos en una perspectiva más amplia, trabada con factores sociales, económicos e ideológicos. Esta amplitud de horizontes trasciende a lo cultural y simbólico y permite responder a muchas de las preguntas que nos hacemos los historiadores del arte. Entre tales cuestiones merece una especial mención el color -sobre el que tanta información aportan las nuevas técnicas de estudio- por sus profundas implicaciones en relación con el valor concedido a la materia. No en vano, la dimensión matérica fue clave en la conceptualización cromática medieval, tal y como se observa en los textos de la época, donde el color comparece descrito en términos de brillantez, pureza o intensidad, normalmente en conexión con objetos o con elementos del mundo natural ${ }^{26}$. Dentro del abanico cromático, las

24 F. TOLAINI, "Trattati tecnici”, en E. CASTELNUOVO y G. SERGI (eds.), Del costruire: techniche, artisti, artigiani, committenti, vol. 2 de Arte e storia nel Medioevo, Turín, 2003, pp. 673-685.

25 Avances producidos en distintas áreas, desde la metalistería a la eboraria, la cerámica, el esmalte o el textil, plasmados en publicaciones y reuniones científicas: P. DANDRIDGE y M.T. WYPYSKI, "Preliminary Technical Study of Medieval Limoges Enamels", P.B. VANDIVER et al. (eds.), Material Issues in Art and Archaeology III, Pittsburgh, 1992, pp. 817-826; R. CARTA (coord.), Arqueometría y arqueología medieval, Granada, 2006; Ivory Analysis Combined - Art History and Natural Science. A Dumbarton Oaks Museum Workshop (24-26 de junio de 2011); y L. RODRÍGUEZ PEINADO y A. CABRERA LAFUENTE (eds.), La investigación textil y los nuevos métodos de estudio, Fundación Lázaro Galdiano, 2014.

26 H. PULliAM, "Color”, N. ROWE (ed.), Medieval Art History Today - Critical Terms, número especial de Studies in Iconography, 33 (2012), p. 4. 
gamas del púrpura gozaron de una especial distinción en el ámbito de lo suntuario, como ilustra en su ensayo Laura Rodríguez a partir de un extenso elenco de soportes y obras entre la Antigüedad Tardía y la Baja Edad Media.

La integración de los aspectos materiales y tecnológicos en el discurso histórico artístico permite ampliar notablemente el cuestionario de partida del investigador y aportar luz a otras problemáticas reorientando el estudio de las obras. En el caso de la cerámica, analizado por Jaume Coll, la transmisión de conocimientos técnicos se entrecruza con la gradación de las esferas sociales que disfrutaban de los productos salidos de los hornos y los repertorios ornamentales y temáticos incorporados. Azucena Hernández muestra a través de piezas tan sofisticadas como los astrolabios que la depuración técnica está al servicio de la precisión requerida por el instrumental científico, pero satisface, asimismo, un determinado nivel de suntuosidad. Este se logra en el bordado estudiado por Ana Cabrera mediante la inclusión de materiales preciosos cuya caracterización requiere reveladores procedimientos analíticos.

Gran parte de los avances en el estudio de las artes suntuarias medievales se ha producido en el marco del museo, depositario de algunas de sus creaciones más sobresalientes. La labor de conservación, restauración y estudio llevada a cabo en las instituciones de acogida redunda en un conocimiento más preciso de los objetos en todas sus dimensiones. Los resultados de esta continua actividad obtienen especial difusión con motivo de las exposiciones. Tales eventos suponen una ocasión para la puesta al día y exploración de nuevas vías en el estudio de obras convertidas por su propio carácter portátil en protagonistas de las muestras, cuyos catálogos se distinguen como auténticas obras de referencia ${ }^{27}$.

Por el diálogo que establecen con el público, los museos desempeñan un papel crucial en la percepción actual de las artes suntuarias medievales. Además, resultan determinantes en el encuadre de estas en los debates académicos, pues ya la mera forma de presentarlas y relacionarlas traduce una línea interpretativa que cala en la praxis histórico-artística ${ }^{28}$. Una de las problemáticas más relevantes en la creación de los discursos expositivos en torno a estas piezas estriba en la descontextualización de las mismas y, a su vez, en los nuevos contextos generados. Esta cuestión es especial-

27 Valga como recordatorio -sin pretensión de exhaustividad- la trascendencia que tuvieron en el ámbito internacional muestras como The Year 1200 (Nueva York, 1970), Rhin-Meuse. Art et Civilisation 800-1400/ Rhein und Maas, Kunst und Kultur, 800-1400 (Colonia, 1972-1973), Ornamenta Ecclesiae. Kunst und Künstler der Romanik (Colonia, 1985), Enamels of Limoges 1100-1350 (París-Nueva York, 1995-1996), Images in Ivory. Precious Objects of the Gothic Age (Detroit-Baltimre, 1997), las celebradas en 2004 en Francia sobre el arte en torno a 1400 -entre otras, Paris 1400. Les arts sous Charles IV (París, 2004)- o las dedicadas al mundo bizantino por el Metropolitan Museum of Art de Nueva York y la Royal Academy of Arts de Londres: The Glory of Byzantium (Nueva York, 1997), Byzantium: Faith and Power (1261-1557) (Nueva York, 2004), Byzantium. 330-1453 (Londres, 2008) y Byzantium and Islam. Age of Transition (Nueva York, 2012). En lo que respecta al arte hispánico, junto a la ya aludida Maravillas de la España Medieval: tesoro sagrado y monarquía, merecen una mención Al-Andalus: The Art of Islamic Spain / Al-Andalus: las artes islámicas en España (Nueva York-Granada, 1992), la no celebrada The Art of Medieval Spain. A.D. 500-1200, cuyo catálogo se publicó en 1993, De Limoges a Silos (Madrid-Bruselas-Santo Domingo de Silos, 2001-2002), y Sancho el Mayor y sus herederos. El linaje que europeizó los reinos hispanos (Pamplona, 2006).

28 M. MARTIN, "Relics of Another Age: Art History, the 'Decorative Arts' and the Museum", Art Bulletin of Victoria, 49 (2010), pp. 7-21. 
mente compleja cuando se enfrenta a la funcionalidad de objetos cuyo uso y entorno originarios quedan diluidos al insertarse en un nuevo marco ${ }^{29}$.

Los museos, como organismos en constante evolución, reflejan en su propio devenir los valores cambiantes asignados también por la historiografía a las artes suntuarias. La profunda reforma acometida por el Museo Arqueológico Nacional en los últimos años ha supuesto una ocasión para replantear el discurso de sus colecciones y su montaje expositivo. Las artes suntuarias medievales ocupan un destacado lugar entre sus fondos, con piezas que se han convertido en iconos de la institución. Ángela Franco nos guía por la renovada instalación a través de sus hitos más significativos en un texto que supone, a la vez, una invitación a contemplarlos con nuevas miradas en sus flamantes salas.

Otra institución cuya renovación museográfica permanece aún en la memoria reciente, el Museo de Telas Medievales del Monasterio de Santa María la Real de Las Huelgas, llevó a cabo su reapertura en 2008. Entre tanto, sus emblemáticas piezas pudieron ser vistas en una exposición en el Palacio Real de Madrid ${ }^{30}$, ocasión propiciada por el acondicionamiento de la sede habitual de las obras que nos remite a experiencias semejantes inmediatas. Ha sido el caso de la célebre serie parisina de la Dama y el Unicornio ${ }^{31}$ y el de los tapices de la Colegiata de Pastrana, restaurados e inmersos en prolongadas giras internacionales mientras se adecuaban sendas instalaciones renovadas para su acogida final. Estos ejemplos, relativos a piezas eminentes, devuelven a la actualidad una movilidad del textil que no es extraña a la propia práctica medieval. Su conservación, exhibición y almacenaje plantean retos que han contado con soluciones diversas a lo largo del tiempo analizadas por Concha Herrero a partir del ejemplo de las colecciones de Patrimonio Nacional.

En su última trayectoria, el Museo Nacional de Artes Decorativas, cuyos fondos nos llevan a sobrepasar los límites del mundo medieval, ha venido experimentado nuevas formas de exponer sus obras. Estas lecturas, atentas a la evolución de los valores sociales, han ido en paralelo a iniciativas que responden a las demandas de sus visitantes y enlazan con otros ámbitos del conocimiento. El texto redactado por Sofía Rodríguez y Paloma Muñoz-Campos, además de trazar la historia centenaria del museo, expone algunos de estos retos y las respuestas -y apuestas- ofrecidas por la práctica museológica llevada a cabo en dicha institución.

Desde su pluralidad de perspectivas, las aportaciones brevemente reseñadas a lo largo de estas páginas enlazan, pues, con las líneas maestras de la reflexión actual en torno a las artes suntuarias, y aspiran a contribuir a los debates aludidos con nuevos argumentos desde el observatorio privilegiado que supone el arte medieval hispano. La variedad y riqueza de sus planteamientos constituyen un estímulo que ha de alentar posteriores investigaciones.

29 J.R. BRANHAM, "Sacrality and Aura in the Museum: Mute Objects and Articulate Space", The Journal of the Walters Art Gallery, 52-53 (1994-1995), pp. 33-47.

30 Vestiduras Ricas. El Monasterio de Las Huelgas y su época. 1170-1340 (16 de marzo - 19 de junio de 2005).

31 Véase el dossier de prensa "La Dame à la Licorne comme vous ne l'avez jamais vue", www.museemoyenage.fr/media/documents-pdf/dossiers-de-presse/dp-dame-la-licorne.pdf (último acceso 25/6/2014). 